\title{
Simplified model for soot generation in a combustion of a biodiesel surrogate
}

\author{
Silvia Barcelos Machado ${ }^{1}$ \\ Graduate Program in Applied Mathematics, UFRGS, Porto Alegre, RS \\ Álvaro Luiz de Bortoli \\ Graduate Program in Applied Mathematics, UFRGS, Porto Alegre, RS \\ Graduate Program in Chemical Engineering, UFRGS, Porto Alegre, RS
}

\begin{abstract}
The objective of this work is to model the soot generation in the combustion of turbulent jet diffusion flame of the biodiesel substitute, methyl decanoate. Fuel oxidation and soot generation were described using a simplified kinetic mechanism based on the HACA mechanism, and the flamelet concept was applied to the temperature and mass fraction equations of the species. Two empirical equations were used to calculate the volume fraction and soot number density, which presented results compatible with the literature.
\end{abstract}

Keywords. CFD; turbulent jet diffusion flame; biodiesel surrogate; methyl decanoate and soot.

\section{Introduction}

The search for clean and renewable energy has been, in recent decades, a subject of global interest, both because of the need to reduce environmental and economic impacts. Studies on different types of energy and their viability have been in focus and in terms of engines, alternatives to gasoline and conventional diesel have been considered. However, the study of combustion and generation of pollutants is still challenging.

Biodiesel, for example, has a highly complex structure that makes it difficult to model [5]. Therefore, biodiesel substitutes, such as methyl decanoate (MD $-\mathrm{C}_{11} \mathrm{H}_{22} \mathrm{O}_{2}$ ) have been widely accepted, due to the simple formulation and combustion characteristics similar to those of biodiesel.

One of the main pollutants generated in the combustion of biodiesel is soot, which is defined as a cluster of carbon microparticles and other solid materials dispersed in the air. Soot particles evolve and change over the course of the burning process and predictions about their spatial distribution, volume fraction and numerical density are important for the development of favorable engine conditions to reduce this pollutant.

In this work, we model the formation and oxidation of soot in the combustion of turbulent diffusive flames from MD. We describe the flamelet concept, the turbulent reactive flow equations and the semi-empirical equations that characterize the stages of soot formation and oxidation. Comparisons are presented and are in accordance with the results of the literature.

\footnotetext{
${ }^{1}$ silvia.machado@ufrgs.br

2 dbortoli@mat.ufrgs.br
} 


\section{Model formulation}

\section{$2.1 \quad$ Flamelet concept}

In turbulent and diffusive combustion processes which one the reaction velocity is faster than the transport phenomena the flamelet concept can be used. In this concept, the turbulent flow field for certain variables is interpreted as a collection of asymptotically thin flames, so they come to be considered as laminar flames. In this way, the turbulence and the chemical part of the combustion process are modeled separately [3].

The flamelet concept is applied to the mass fraction equations of the chemical species involved in combustion and to the temperature, becoming one-dimensional and time-dependent equations [7]. That is, a coordinate transformation is done, applied to the flame surface, with a new $\mathrm{Z}$ coordinate and time $\tau$. The variable $\mathrm{Z}$ represents the fraction of the fuel-oxidant mixture.

Considering realistic situations, treating finite chemical reaction rate and fluctuations in the flow field, the temperature and mass fraction of species equations are written as [7]:

$$
\frac{\partial \Psi}{\partial \tau}=\frac{\chi}{2} \frac{\partial^{2} \Psi}{\partial Z^{2}}+\omega_{\Psi}
$$

where $\Psi$ represents the temperature and mass fraction of species, $\chi$ is the scalar dissipation rate, $\dot{\omega}_{\Psi}$ is the source term.

The scalar dissipation rate is associated with the convection and diffusion effects of the mixing process and it is expressed as $\chi=2 D(\nabla Z)^{2}$, where $D=D_{Z}$, or $D=D_{k}$, or $D=D_{T}$ is the molar diffusivity of $\mathrm{Z}$, mass diffusivity of the species $k$ or thermal diffusivity, respectively. The scalar dissipation rate is directly involved to the system turbulence, since the greater the turbulence, the greater the fraction gradient of the mixture and, therefore, the greater the scalar dissipation rate. Peters (1984) approximated the scalar dissipation rate using the error function [7]:

$$
\chi=\chi_{s t} \frac{\exp \left\{-2\left[\operatorname{erfc} c^{-1}(2 Z)\right]^{2}\right\}}{\exp \left\{-2\left[\operatorname{erfc} c^{-1}\left(2 Z_{s t}\right)\right]^{2}\right\}} ;
$$

where $\chi_{s t}$ is the stoichiometric scalar dissipation rate, taken equal to $5 / s$ and 10/s [10].

If we consider global mechanisms, mainly the one-step mechanism, and a high Damköhler number, which means taking the chemical reaction infinitely fast, there is an approximate solution to the Burke-Schumann solution for the main variables [8]:

For $Z<Z_{s t}$ :

$$
\left\{\begin{array}{l}
Y_{F}=0 \\
Y_{O_{2}}=Y_{O_{2}, 2}\left(1-\frac{Z}{Z_{s t}}\right) \\
T=T_{1}+Q_{1} Z
\end{array}\right.
$$

For $Z \geq Z_{s t}$ :

$$
\left\{\begin{array}{l}
Y_{F}=Y_{F, 1}\left(\frac{Z-Z_{s t}}{1-Z_{s t}}\right) \\
Y_{O_{2}}=0 \\
T=T_{1}+Q_{2}(1-Z)
\end{array}\right.
$$

where $Y_{F}$ is the mass fraction of the fuel, $Y_{O_{2}}$ is the mass fraction of the oxidant, $T$ is the temperature, $Z_{s t}=\frac{Y_{O_{2}, 2}}{\nu Y_{F, 1}+Y_{O_{2}, 2}}$ is the stoichiometric mixture fraction, $Y_{O_{2}, 2}$ and $Y_{F, 1}$ are the mass fractions of the oxidizer and fuel in the free stream, respectively; $T_{1}$ is the initial temperature, $Q_{1}$ and $Q_{2}$ are parameters to the adiabatic temperature of the flame. 


\subsection{Turbulent flow equations}

The other variables involved in the problem are expressed using the complete Navier-Stokes equations and to model the turbulence, which encompasses several scales, numerical procedures such as the LES (Largy Eddy Simulation) technique are applied. The LES technique consists of applying a spatial filter [9] to conservation equations, whose variables are rewritten as the sum of two terms: one of average values, which represents large-scale movements and they are solved numerically; and a floating-value term, which represents small-scale movements and they are modeled.

In the LES technique, the Reynolds and Favre averages [3] are used, presented respectively in the equations (5) and (6). The Favre average is composed by a density weighted average and it is indicated for modeling compressible flows with large variations in density.

$$
\Phi=\bar{\Phi}+\Phi^{\prime} ; \text { with } \overline{\Phi^{\prime}}=0 \text { and } \bar{\Phi}=\lim _{\Delta t \rightarrow+\infty}\left(\frac{1}{\Delta t} \int_{t_{0}}^{t_{0}+\Delta t} \Phi(t) d t\right) ;
$$

and

$$
\Phi=\tilde{\Phi}+\Phi^{\prime \prime} ; \text { with } \rho \overline{\Phi^{\prime \prime}}=0 \text { and } \tilde{\Phi}=\frac{\overline{\rho \Phi}}{\bar{\rho}}=\lim _{\Delta t \rightarrow+\infty}\left(\frac{\int_{t}^{t+\Delta t} \rho \Phi(t) d t}{\int_{t}^{t+\Delta t} \rho d t}\right) .
$$

Using the described averages, the equations for reactive turbulent flow are obtained:

$$
\begin{aligned}
& \frac{\partial \bar{\rho}}{\partial t}+\frac{\partial\left(\bar{\rho} \widetilde{u_{j}}\right)}{\partial x_{j}}=0 ; \\
& \bar{\rho} \frac{\partial \widetilde{u_{i}}}{\partial t}+\bar{\rho} \widetilde{u_{j}} \frac{\partial \widetilde{u_{i}}}{\partial x_{j}}=-\frac{\partial \widetilde{p}}{\partial x_{i}}+\frac{\partial \overline{\phi_{i j}}}{\partial x_{j}}-\frac{\partial\left(\bar{\rho} \widetilde{\left.u_{j}^{\prime \prime} u_{i}^{\prime \prime}\right)}\right.}{\partial x_{j}} ; \\
& \bar{\rho} \frac{\partial \widetilde{Z}}{\partial t}+\bar{\rho} \widetilde{u_{j}} \frac{\partial \widetilde{Z}}{\partial x_{j}}=\frac{\partial}{\partial x_{j}}\left(\overline{\rho D} \frac{\partial \widetilde{Z}}{\partial x_{j}}\right)-\frac{\partial\left(\bar{\rho} \widetilde{u_{j}^{\prime \prime} Z^{\prime \prime}}\right)}{\partial x_{j}} ; \\
& \nabla^{2} \bar{p}=\Delta t\left(\frac{\partial \bar{\rho}}{\partial t}+\frac{\partial\left(\bar{\rho} \widetilde{u_{j}}\right)}{\partial x_{j}}\right) ; \\
& \bar{\rho} \frac{\partial \widetilde{Y_{k}}}{\partial \tau}=\bar{\rho} \frac{\widetilde{\chi}}{2} \frac{\partial^{2} \widetilde{Y_{k}}}{\partial Z^{2}}+\widetilde{\omega_{k}} ; \\
& \bar{\rho} \frac{\partial \widetilde{T}}{\partial \tau}=\bar{\rho} \frac{\tilde{\chi}}{2} \frac{\partial^{2} \widetilde{T}}{\partial Z^{2}}+\widetilde{\omega_{T}} ;
\end{aligned}
$$

where $\rho$ is the density, $u_{i}$ is the velocity component in the direction $i, p$ is the pressure, $\phi_{i j}$ is the viscous stress tensor component and $Y_{k}$ is the mass fraction of the species $k$.

The Reynolds tensor, $\bar{\rho} u_{j}^{\prime \prime} u_{i}^{\prime \prime}$, represents the tension exercised by fluctuations in the turbulent flow. This term can be approximated by the velocity gradient $\bar{\rho} \widetilde{u_{j}^{\prime \prime} u_{i}^{\prime \prime}}=-\bar{\rho} \mu_{T} \frac{\partial \widetilde{u}}{\partial y}$, where $\mu_{T}$ is the turbulent viscosity, an artificial parameter that represents the resistance associated with flow.

The Agrawal-Prasad model [1] proposes an analytical solution for the turbulent viscosity in turbulent axisymmetric jet, given in the form:

$$
\mu_{T}=U_{c} L c \frac{\sqrt{\pi}}{8} \frac{\operatorname{erf}(\xi)}{\xi} ; \xi=\frac{r}{c x}
$$

where $U_{c} \approx x^{-1 / 2}$ is the velocity profile on the jet central line, $L \approx x$ is the characteristic length, $c \simeq 0.107$ is the Agrawal-Prasad coefficient and $r$ is the radius of the axisymmetric jet. 


\subsection{Soot formation and oxidation}

Soot precursors, polycyclic aromatic hydrocarbons (PAHs), usually are formed during the process of incomplete combustion of hydrocarbon-rich fuels, under high temperatures, near to the stoichiometric region of the flame.

Soot formation can be described by the following steps [2]: inception, particle growth, coagulation and oxidation. In the first step, the fuel molecules break and the formation of PAHs and soot begins; in the second, the soot particle nucleus grow up and the particles earn mass and volume, forming larger particles; during coagulation, the particles agglomerate attaching with each other; and finally, in oxidation stage, the particles can be oxidized for $\mathrm{O}_{2}$ or $\mathrm{OH}$, removing carbon atoms from the particle and, in some cases, fragmenting the particle. This last step is important to reduce the size of soot.

The hydrogen abstraction carbon addition (HACA) [2] mechanism has been used to describe the formation of soot. In several studies, this mechanism was combined with reduced or skeletal mechanisms for hydrocarbons and their surrogates, in order to predict soot generation. Although the use of simplified mechanisms reduces the precision of the results of soot generation, the advantage of the lower computational cost stands out.

In this context, the soot generation prediction from empirical and semi-empirical models also contributes to the reduction of computational costs and, even so, presents reasonable precision. The semi-empirical model presented by Cai et al. (2016) [4] is composed of two equations that describe the volume fraction $\left(\rho Y_{s}\right)$ and the soot number density $\left(N_{s}\right)$ and, including the steps of inception, growth, coagulation and soot oxidation. The general form of the equations are:

$$
\begin{aligned}
& \frac{d\left(\rho Y_{s}\right)}{d t}=\alpha_{1}+\alpha_{2}-\alpha_{3}-\alpha_{4}, \\
& \frac{d\left(N_{s}\right)}{d t}=\beta_{1}-\beta_{2},
\end{aligned}
$$

where $\alpha_{1}, \alpha_{2}, \alpha_{3}, \alpha_{4}, \beta_{1}$ e $\beta_{2}$ are source terms defined below.

As the species $\mathrm{C}_{2} \mathrm{H}_{3}$ directly influences the soot inception [4], it is written as:

$$
\begin{aligned}
\alpha_{1} & =L_{\alpha_{1}} \times \exp \left(-E_{\alpha_{1}} / T\right) \times \rho \times Y_{C_{2} H_{3}}, \\
\beta_{1} & =\frac{\alpha_{1}}{W_{C_{2} H_{3}} \times C_{n}},
\end{aligned}
$$

in which $W_{C_{2} H_{3}}$ is the molecular mass of $C_{2} H_{3}$ and $C_{n}=12$ is the number of carbon atoms in the initial soot particle.

For the particle growth, it is considered that acetylene, $\mathrm{C}_{2} \mathrm{H}_{2}$, is the main species to attach the others, thus contributing to the growth of the soot surface. The equation for the source term for this step is as follows:

$$
\alpha_{2}=L_{\alpha_{2}} \times \exp \left(-E_{\alpha_{2}} / T\right) \times \sqrt{S} \times \rho \times Y_{C_{2} H_{2}},
$$

where $S$ is the soot surface area, given by:

$$
S=\pi N_{s}\left(\frac{6 \rho Y_{s}}{\pi \rho_{s} N_{s}}\right)^{2 / 3},
$$

in which $\rho_{s}=1,8 \mathrm{gcm}^{-3}$ is the density of the soot particle.

Coagulation reduces the number of soot particles, thus influencing only in equation (15). So, 


$$
\beta_{2}=2,25 \times 10^{15} \times T^{1 / 2} \times N_{s} .
$$

In contrast to the soot particle growth, oxidation occurs through two routes: oxidation by $\mathrm{O}_{2}$ or by the radical $\mathrm{OH}$. Since MD is an oxygenated hydrocarbon, greater amounts of $\mathrm{O}_{2}$ and $\mathrm{OH}$ are expected and this directly influences oxidation and also indirectly reduces the amount of $\mathrm{C}_{2} \mathrm{H}_{3}$. This is one of the factors for the production of soot in biodiesel to be lower than in conventional diesel.

The oxidation by $\mathrm{O}_{2}$ is modeled by the equation:

$$
\alpha_{3}=L_{\alpha_{3}} \times \frac{12 \times S}{M_{s}}\left(\frac{K_{A} P_{O_{2}}}{1+K Z P_{O_{2}}} x+K_{B} P_{O_{2}}(1-x)\right),
$$

where $M_{s}$ is the molecular mass of one carbon atom, $P_{\mathrm{O}_{2}}$ is the partial pressure of oxygen and

$$
\begin{aligned}
x & =\frac{P_{O_{2}}}{P_{O_{2}}+K_{t} / K_{B}}, \\
K_{A} & =30 \times \exp (-15800 / T), \quad K_{B}=8 \times 10^{-3} \times \exp (-7640 / T), \\
K_{T} & =1,51 \times 10^{5} \times \exp (-49800 / T), \quad K_{Z}=27 \times \exp (3000 / T) .
\end{aligned}
$$

The oxidation by $O H$ is expressed as:

$$
\alpha_{4}=L_{\alpha_{4}} \times \gamma_{O H} \times \chi_{O H} \times T^{-1 / 2} \times S \times \exp (-19023 / T),
$$

where $\gamma_{O H}=0,1$ represents the collision efficiency between the soot particle and the radical $O H$ and $\chi_{O H}$ is the molar fraction of $O H$.

The parameters $L_{\alpha_{1}}, L_{\alpha_{2}}, L_{\alpha_{3}}, L_{\alpha_{4}}, E_{\alpha_{1}}$ and $E_{\alpha_{2}}$ are chosen from a sensitivity study developed in [4]. Variation of these parameters softens or accentuates the peak soot volume fraction, and some combinations of parameters significantly alter the volume fraction gradients.

\section{$3 \quad$ Numerical results}

A simplified mechanism of oxidation of the MD and the main reactions of the HACA mechanism involving acetylene and $\mathrm{C}_{2} \mathrm{H}_{3}$ were coupled. The equations were discretized by the finite difference method and solved by Rosenbrock method [3] for turbulent jet diffusion flame. The burner has a $10 \times 1 u$ domain in the axial and radial directions. The nozzle length is unitary and its diameter is $0.1 u$. The mesh has $251 \times 51$ vertices and it is refined in the region close to the jet nozzle.

The mass fractions of $\mathrm{C}_{2} \mathrm{H}_{2}$ and $\mathrm{C}_{2} \mathrm{H}_{3}$ were compared with the data presented in [4] and are shown in the figures (1) and (2). These species are essential for the soot formation. The last figure also shows the mass fraction of the radical $O H$, important for soot oxidation.

The flamelet equations for soot volume fraction and soot density number were solved considering the optimized parameters described in the table (1). The fuel considered in this work is a biodiesel surrogate (MD) pure. In [4], a mixture of 25\% MD, 25\% methyl-9-decanoate (MD9D) and $50 \%$ n-heptane was used; then, for the comparison, the stoichiometric region was slightly shifted to the right to coincide with the stoichiometric region related to the combustion of the trio of biodiesel surrogates. The simplified model predicted larger gradients of soot volume fraction in the stoichiometric region of the flame as well as in [4], overestimating this value by $\approx 3.7 \times 10^{-5}$. This increase is expected when a reduced number of reactions is used to model combustion and soot generation. 
Table 1: Optimized parameters of the two equation model.

\begin{tabular}{c|c|c|c|c|c|c}
\hline$\chi_{s t}$ & $L_{\alpha_{1}}$ & $E_{\alpha_{1}}$ & $L_{\alpha_{2}}$ & $E_{\alpha_{2}}$ & $L_{\alpha_{3}}$ & $L_{\alpha_{4}}$ \\
\hline 5 & 625 & 10000 & $3,0 \times 10^{11}$ & 40000 & 200 & $8,15 \times 10^{13}$ \\
\hline
\end{tabular}

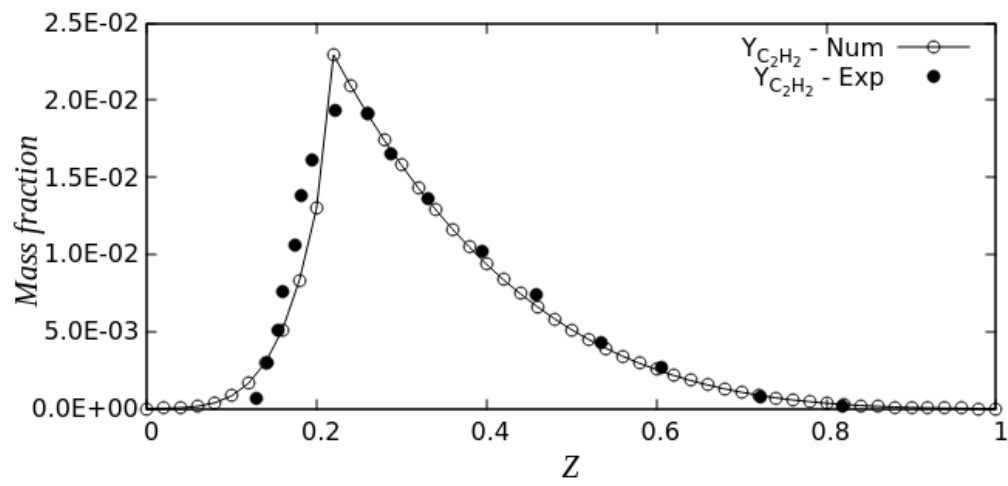

Figure 1: Mass fraction of acetylene.

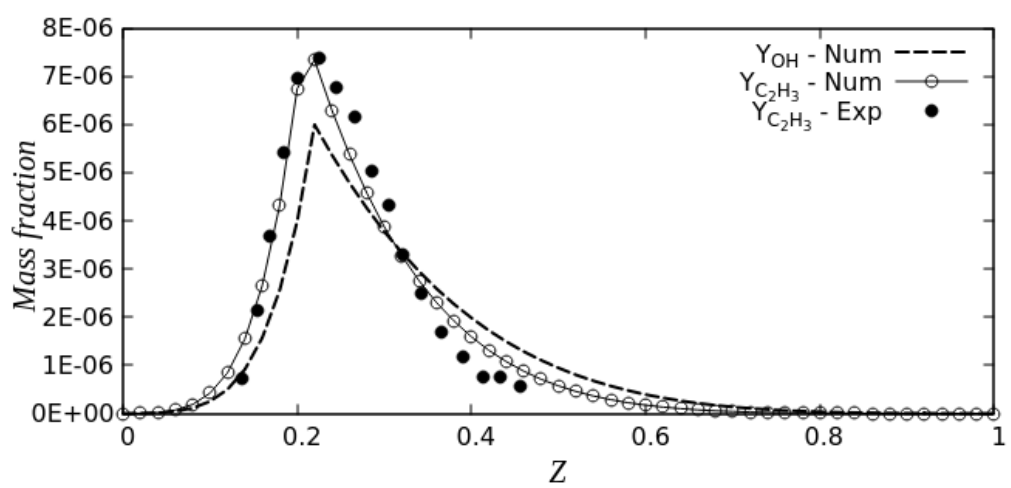

Figure 2: Mass fraction of $\mathrm{C}_{2} \mathrm{H}_{3}$ and $\mathrm{OH}$.

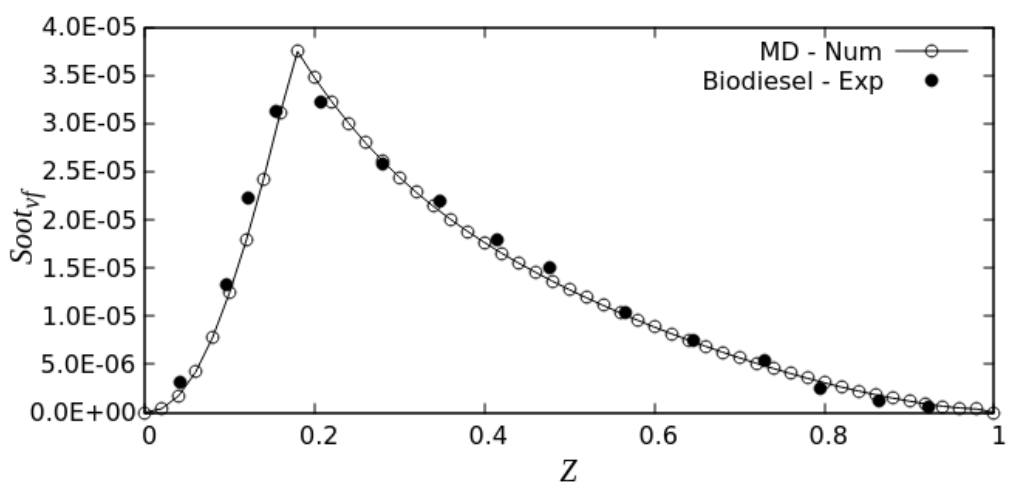

Figure 3: Volume fraction of soot in the combustion of the MD. 


\section{Conclusions}

In [4], a 115 species reduced mechanism was used for a mixture of three biodiesel surrogates. In this work, we consider a 25 species simplified mechanism for a single biodiesel surrogate: the MD. The use of simplified models is useful for a first study of soot generation in the combustion of biofuels, since the computational cost is small and the results obtained, although overestimating some values, are in agreement with those obtained in the literature, obtained numerically and experimentally.

Although the model is simplified, it considers different steps for the formation and oxidation of soot, addressing all the important characteristics of soot generation and being able to predict the peak regions of fraction of soot volume.

\section{Acknowledgment}

This research is being developed at UFRGS. The first and second authors gratefully acknowledge the financial support from CAPES, Coordenação de Aperfeiçoamento de Pessoal de Nível Superior, whereas Prof. De Bortoli acknowledges the financial support from CNPq, Conselho Nacional de Desenvolvimento Científico e Tecnológico under grant 306768/2018-6.

\section{References}

[1] Agrawal, A. and Prasad, A. K. Integral solution for the mean flow profiles of turbulent jets, plumes, and wakes, J. Fluids Eng., volume 125(5):813-822, 2003. DOI: 10.1115/1.1603303.

[2] Appel, J., Bockhorn, H. and Frenklach, M. Kinetic modeling of soot formation with detailed chemistry and physics: laminar premixed flames of $\mathrm{C} 2$ hydrocarbons, Combustion and Flame, volume 121:122-136, 2000. DOI: 10.1016/S0010-2180(99)00135-2.

[3] Bortoli, A. L., Andreis, G. S. L. and Pereira, F. N. Modeling and Simulation of Reactive Flows. Elsevier, 2015. ISBN: 978-0-12-802974-9.

[4] Cai, G., Yen, M. and Abraham, J. On formulating a simplified soot model for diesel and biodiesel combustion, Chemical Engineering Science, volume 144:249-259, 2016. DOI: 10.1016/j.ces.2016.01.039.

[5] Lai, J. Y. W., Lin, K. C. and Violi, A. Biodiesel combustion: Advances in chemical kinetic modeling, Progress in Energy and Combustion Science, volume 37:1-14, 2011. DOI: $10.1016 / \mathrm{j} \cdot$ pecs.2010.03.001.

[6] Lele, A. D., Anand, K., and Narayanaswamy, K. Surrogates for Biodiesel: Review and Challenges. Springer, 2017. ISBN: 978-981-10-3790-0.

[7] Peters, N. Laminar diffusion flamelet models in non-premixed turbulent combustion, Combust. Sci. Technol, volume 10(3):319-339, 1984. DOI: 10.1016/0360-1285(84)90114-X.

[8] Peters, N. Fifteen lectures on laminar and turburlent combustion. Germany: Ercoftac Summer School, 1992.

[9] Peters, N. Turbulent Combustion. Cambridge University Press, 2000.

[10] Venugopal, R. and Abraham, J. Unsteady flamelet response in the near field of High-ReynoldsNumber jets, AIAA Journal, volume 47(6):1491-1506, 2009. DOI: 10.2514/1.40153. 